\title{
Combined dietary magnesium and potassium intake is associated with greater bone density in women in the EPIC-Norfolk cohort
}

\author{
R. P. G. Hayhoe ${ }^{1}$, M. A. H. Lentjes ${ }^{2}$, R. N. Luben ${ }^{2}$, K-T. Khaw ${ }^{2}$ and A. A. Welch ${ }^{1}$ \\ ${ }^{1}$ Population Health and Primary Care, Norwich Medical School, University of East Anglia, Norwich, Norfolk NR4 \\ $7 T J$ and ${ }^{2}$ Public Health and Primary Care, University of Cambridge, Cambridgeshire CB1 8RN
}

A multitude of factors are known to influence bone health, including modifiable factors such as diet, physical activity, and smoking, but also determinants such as age, sex, and genetics ${ }^{(1)}$. Calcium and vitamin D have traditionally been the key nutritional candidates for osteoporosis prevention and maintenance of bone health ${ }^{(2)}$. Recently, evidence has been emerging linking magnesium and potassium intakes with bone mass, and their deficiency with osteoporosis ${ }^{(2,3,4)}$. The current study therefore aimed to explore the potential association of dietary magnesium and potassium intake with bone density status in a UK population.

A random sample of 4000 individuals from the approximately 25,000 participants in the EPIC-Norfolk cohort ${ }^{(5)}$ was used for analysis. The Norfolk District Health Authority Ethics Committee approved all procedures. Participants completed a selfadministered health and lifestyle questionnaire and dietary intake was estimated using 7-day food diaries. Quantitative ultrasound measurements of the calcaneus were performed according to standardised protocols using a CUBA device (McCue Ultrasonics, Winchester), giving measurements of ultrasound attenuation (BUA) and velocity of sound (VOS).

Multivariate regression analysis was used to investigate differences in calcaneal BUA and VOS across sex-specific quintiles of magnesium+ potassium dietary intake $\mathrm{z}$ scores. The combination $\mathrm{z}$ score variable was created by standardising summed $\mathrm{z}$ scores of magnesium and potassium intake ${ }^{(3)}$, necessary due to the significant collinearity evident between these nutrients. The full model was adjusted for age, body mass index (BMI), smoking status, physical activity, family history of osteoporosis, menopausal and hormone replacement therapy (HRT) status in women, calcium intake, and total energy intake. Statistical analyses were performed using STATA (v12; Stata Corp., USA).

There were 968 men and 1360 women with data for all selected variables. Mean (SD) age was 63.0 (9.3) for men and 61.7 (9.2) for women. The majority (75.4\%) of women were post-menopausal and 35.3\% were current or former users of HRT. Mean magnesium intake was 3-10\% higher than the UK Reference Nutrient Intake (RNI) (300 mg men; $270 \mathrm{mg}$ women) ${ }^{(6)}$. Mean potassium intake in women was $12 \%$ lower than the RNI $(3500 \mathrm{mg})^{(6)}$. No significant trends in BUA or VOS were apparent across quintiles of magnesium + potassium z scores for men, but for women there were significant trends for BUA in both the unadjusted $(p<0 \cdot 01)$ and full model $(p=0.03)$, and VOS in the unadjusted model $(p=0.04)$. Fully adjusted BUA was $5.5 \%$ greater in quintile 5 versus quintile 1 for women and $0.9 \%$ greater for men.

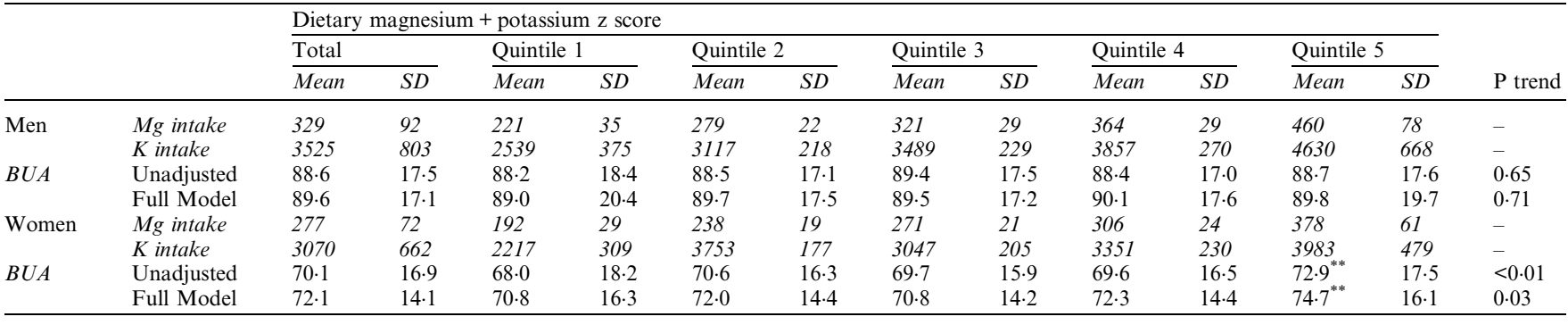

** $p<0.01$ versus quintile 1 . Magnesium $(\mathrm{Mg})$ and Potassium $(\mathrm{K})$ intakes in $\mathrm{mg} /$ day. BUA in $\mathrm{dB} / \mathrm{MHz}$.

In this study combined dietary magnesium and potassium intake has been positively associated with a quantitative measure of bone density. These results complement the limited literature studying other populations ${ }^{(2,3,4)}$, presenting UK-relevant data and demonstrating that further investigation is warranted into the mechanisms of this association and the potential protective role of magnesium and potassium against osteoporosis.

1. Martini LA (1999) Nutr Rev 57, 227-9.

2. Rondanelli M, Opizzi A, Perna S, et al. (2013) Endocrinol Nutr 60, 197-210.

3. Tucker KL, Hannan MT, Chen H, et al. (1999) Am J Clin Nutr 69, 727-36.

4. New SA, Robins SP, Campbell MK, et al. (2000) Am J Clin Nutr 71, 142-51.

5. Day N, Oakes S, Luben R, et al. (1999) Br J Cancer 80, 95-103.

6. Department of Health (1991) Report on Health and Social Subjects 41. 